\title{
CORRIGENDUM TO: SMOOTH NONTRIVIAL 4-DIMENSIONAL $s$-COBORDISMS
}

\author{
SYLVAIN E. CAPPELL AND JULIUS L. SHANESON
}

In [1] we constructed a family of nontrivial topological s-cobordisms of 3dimensional quaternionic spaces. This and further considerations led to the result that there are either $2^{2^{r}-r-1}$ or $2^{2^{r}-r}$ distinct $s$-cobordisms of any quaternionic space-form $M_{r}=S^{3} / Q_{r}$ to itself, where $Q_{r}$ denotes the quaternion group of order $2^{r+2}$. In [2] we erroneously claimed, using in part various exact sequences in algebraic $L$-theory, that the upper bound was precise, and used this to detect the topological nontriviality of some explicitly constructed smooth $s$-cobordisms. Reconsideration of this material using some exact sequences of Ranicki [4] and particularly the related unpublished work on algebraic "visible" $L$-theory of Michael Weiss [5] leads to the opposite conclusion:

THEOREM. There are precisely $2^{2^{r}-r-1}$ topologically distinct $s$-cobordisms of the quaternionic space $M_{r}^{3}$ to itself.

In particular, the questions of whether the construction of [2] is smoothly a product, as well as the smoothability of the above examples, remain open. The above theorem will be proved in [3].

\section{BIBLIOGRAPHY}

1. S. Cappell and J. L. Shaneson, On 4-dimensional s-cobordisms, J. Differential Geom. 22 (1985), 97-115.

2. 141-143.

3. __ On 4-dimensional s-cobordisms. II (to appear).

4. A. Ranicki, The algebraic theory of surgery. I, II, Proc. London Math. Soc. (3) 40 (1980), 87-283.

5. M. Weiss, On the definition of the symmetric L-groups (to appear).

Department of Mathematics, Courant Institute, New York UniverSITY, NEW YORK, NEW YORK 10012

Department of MAThematics, RUtgers University, NeW BRUnSWick, NEW JERSEY 08903

Received by the editors July 28, 1987.

1980 Mathematics Subject Classification (1985 Revision). Primary 57R80.

Both authors partially supported by NSF grants. 
